\title{
Research on the Modulation Mode of Wireless Optical Communication Technology
}

\author{
WANG Juan \\ Chongqing College Of Electronic Engineering,Chongqing,China \\ wj8888886@126.com
}

Keywords: Wireless Optical, Communication Technology, Modulation Mode

\begin{abstract}
In recent years, with the development of key components of optical communications, wireless optical communication technology is developing rapidly, and gets a lot of attention and research. Wireless optical communication technology is superior to traditional radio communications technology, in that it has its own advantages: wireless optical communication has a wealth of free spectrum, from infrared to ultraviolet, can provide large amounts of band communication; it is hardly affected by emitted electromagnetic blessing, does not interfere with other sensitive electromagnetic devices, has good transmission of information confidentiality; less communication device volume, easy to carry, without geographical factors; low power consumption, for a variety of different bands Select the transmission of information. Thus, a Subsidiary of wireless optical communication has a strong practical value.
\end{abstract}

\section{Introduction}

Nearly two decades of explosive growth in the popularity of mobile communications and the Internet so that people can enjoy all kinds of mobile Internet applications and convenient information, entertainment and consumption anytime, anywhere through a wireless network. The number of users with the development of new technology continues to climb on high, at the same time, communication spectrum and the growing shortage of rental price up to more than ten billion, .IP address the increasing depletion of resources [1]. To alleviate this situation, we need a way to make up for the traditional radio communication spectrum resource constraints, or even replace it new technology. FSO is a light as an information carrier, the use of free space as a new technology channel signal transmission. It not only has the traditional characteristics of wireless communication, but also combines the characteristics of the emerging optical communication, such as high bandwidth, low blessing shot, low energy consumption and equipment from electromagnetic interference and other characteristics [2]. With the maturity of optical communication technology and the continuous development of LED and other optical devices, optical communication in wireless bandwidth, performance, reliability, and improve, and therefore has been widespread concern in the domestic and international research institutions, will also play in the future of wireless communications important role. In this thesis, from a technical point of view, the use of channel coding performance of wireless optical communications research conducted traitor, by combining with the traditional modulation techniques based on new technologies of wireless optical communications.

\section{The Wireless Optical Communication Theory}

Wireless optical communication system combines the advantages of wireless communication to communicate with fiber, not only can wirelessly communicate freely, but also has high-speed and reliable. The basic structure of a wireless optical communication by the sender, and the receiver channel, of which the channel is a wireless optical channel. Transmission side input source, modulator, drive, and a light source and a corresponding optical system. By the receiver detector, an amplifier, a demodulator and a corresponding optical system.

Wireless optical communication systems, depending on the mode of operation can be divided into quasi-sight communications and line of sight communication. The-horizon communications 
rely mainly on the two sides aligned communication transceiver, channel loss is small and the need to consider the multipath effect, it has a relatively large capacity. Quasi-horizon communications rely mainly on photon scattering signal transmission, no need to send and receive sides aligned, but which bring more severe multipath effects and channel loss. Contrast the above two methods of work, a strong quasi-horizon communications mobility and flexibility than the line of sight communication. According to the operating wavelength range can be divided into ultraviolet light, visible and infrared light.

UV Communication and Its Characteristics. Ultraviolet spectral distribution in the wavelength range of $100 \mathrm{~nm}$ to $380 \mathrm{~nm}$ band. Concrete can be divided into vacuum ultraviolet (100-200nni), far ultraviolet C-band (200nm-280), ultraviolet B band (280nm-315nm) and near ultraviolet A band (315nm-380nm).

Compared to other bands, wherein a wavelength between 200nm and 280nm of C-band called "blind date." The ozone layer of the atmosphere C-band ultraviolet absorption jurisdiction emitted strong, so that the ultraviolet band in the near-Earth atmosphere is almost non-existent, so the band shot a blessing from the sun influence the formation of a so-called blind date, so the band ultraviolet light ultraviolet light communication to provide protection. First, the ozone layer would be the C-band ultraviolet light absorption, even if the communication in the outdoor environment, surface in a faint background noise is also difficult to produce the C-band signal interference. Second, the dust in the air, aerosols and other particles cause scattering of the band of ultraviolet light, which makes the sending and receiving end equipment can be carried out without strict alignment of communication. However, it is the strong scattering of dust in the air, aerosol particles and the UV band is also subject to certain atmospheric absorption, signal attenuation is very large, the signal transmission distance is severely limited. UV Communication has the following characteristics: (1) quasi-sight transmission mode, the atmosphere in a variety of particle scattering intensity characteristic of ultraviolet light so that it can communicate across all kinds of obstacles. (2) local area communication, because of its ultraviolet light scattering characteristics of the band path loss is very serious, so that by controlling the transmission power or other technical means to limit the scope of the communication in the region hope.

The Visible Light Communication and Its Characteristics. Common spectrum distribution of $400 \mathrm{~nm}$ to $700 \mathrm{~nm}$ wavelength of visible light band, but also for different chromatographic light purple wherein the shortest wavelength and red the longest wavelength. It is used to communicate to consider two factors, the first is a large enough power to keep the lights can both communicate; second is due to the sunlight, the band is used when communicating with noisy background. Therefore, visible light communication system more suitable for small indoor environment background noise.

With the luminous efficiency, LED display has been extended to the field from a professional lighting areas of daily life. White LED low power consumption and long life, environmental protection and many other advantages that it can replace the traditional lighting of a new generation light source. In addition, LED for fast modulation characteristics of high-speed communication can be guaranteed by flash W sparkle. And compared to other means of communication, visible light communication technology has the following unique features: (1) human security. Visible light communication system using LED as a source to send data, the low-band electromagnetic blessing shot, no harm to the human eye. (2) high signal to noise ratio. In order to take into account the effect of lighting, interior white LED lighting systems typically use an LED array, which can reach tens of watts of power or more, visible light communication system has a very high signal to noise ratio to ensure good communication quality. (3) everywhere. Visible light communication system used to communicate with its scattering effect, less demanding on the directivity. So long as there are places available using visible light communication, coverage is good. (4) visible light communication does not take existing spectrum resources without authorization can be used flexibly. (5) LED communication speed can reach tens or even several Mbps Mbps, so that it can achieve Gbps communication rate of the order of high order modulation techniques. 


\section{The UV Communication Modem Technology}

The need to reduce the error rate of wireless optical communication system, improve the performance of the entire communication system. To use ultraviolet light to communicate over short distances NLOS communications require higher transmit power to overcome the light attenuation of the signal, but the high power UV light on the human body, especially the human eye injury is very serious, it is not blind to increase Great light emitted optical power; photo detectors by increasing the detection area, so that the light signal energy received increases, but a large area photo detector will cause the receiving end of the capacitor increases communication bandwidth narrower bandwidth efficiency decreased; can improve the signal to noise ratio of the receiver, you can use high-power lasers, high-sensitivity laser detectors and laser wavelength selection for atmospheric window, in addition to the sensitivity of the receiver device technology has also been limited, increase the sensitivity of a single receiver or not completely solve the bad weather wireless optical communication problems; before you can use forward error correction coding techniques, you can also take different modulation methods to improve the reliability and effectiveness of the system, therefore, the transmission of the original information codec technology and signal modulation demodulation of wireless application technology to enhance the overall performance of optical communication plays a big role. In the current situation and the development of existing optical devices based on the need to reduce energy consumption as much as possible on a mobile device, searching for UV Communication modem technology to improve the performance of UV Communication is very important. So the wireless optical communication system for digital signal processing section is relatively high.

Wireless optical modulation technique refers to an information-carrying signal loaded on the light, the control parameters of a light wave, making it as the law of the modulation signal and the process of change; demodulation technique refers to the received optical path reduced to the sender and effective process of information signals containing useful information is converted into an electrical signal by the photo detector. There are many optical modulation method from the modulation method can be divided into direct and indirect modulation. Indirect modulation of better performance, but its structure is complicated and therefore more direct modulation scheme in a wireless optical communication, including direct modulation FM modulation and AM modulation of two common modulations. Since the UV transmission will be absorbed in the atmosphere and scattering properties, and because of the nonlinear electro-optical power conversion process, and therefore better than using FM modulation amplitude modulation, pulse better than the sine wave, the system selects the FM modulator pulse.

Off keying modulation, referred to as OOK, wireless optical communication system is commonly used in the direct pulse intensity modulated by switching the light source to achieve, send a message to 1 , the light source transmitting light, send a message to 0 , the light source does not emit light.

Pulse position modulation. Pulse position modulation, referred to as PPM, a pulse signal is adjusted according to changes in the position of the modulation method varies. It is thus M-bit binary data is mapped from the clock periods through information L-PPM modulated pulse signal is only a single clock cycle, which transmitted information bits as log2M, and L- PPM digit code group is fixed, wherein there is a one clock cycle, clock cycle 0 the other.

A number of differential pulse position modulation L-DPPM slot cycle is not fixed code group, each code group is made after a series of low level to a high level slot cycle plus time slot period constituted. Differential pulse position modulation PPM DPPM simply modulated signal high low clock cycles later removed to complete the encoding, which is a pulse position modulation PPM modulation improved.

DPIM pulse interval modulation is carried out in DPPM modulation on the basis of an improved modulation scheme, similar to DPPM, the median its code group is not fixed. DPIM code group can be divided into two kinds, one is unprotected slot, the other one is a guard slot. To minimize the effects of inter symbol interference by a communication system, and most of us have a guard slot to adopt the DPM modulation, the modulation code group SK, where k represents a decimal number, 
the first bit of information per symbol It is a pulse signal, and then followed by a guard slot, the empty slots plus $\mathrm{k}$, which represents a symbol information. In each symbol received by the receiver when the information does not need to extract the synchronized clock, the symbol synchronization is not required, to give just a symbol information representing the number of slots based on the received pulses contained in the back of the digital subtracting i.e. can. The large degree of modulation method can simplify the complexity of the whole communication system.

\section{The Several Wireless Optical Modulation Average Transmitted Power Performance Analysis}

These four kinds of wireless light emitted optical power modulation comparative analysis below.

The On-Off Keying Modulation OOK . For non-zero on-off keying modulation OOK, when the light source is assumed that sent the original message "1" when the light-emitting power of P1, under effective when information transmitted contains the same probability of " 0 " and " 1 ", the entire modulation the average light power technology is

$$
P_{\text {ook }}=P_{1} / 2
$$

Pulse Position Modulation $P P M$. In $P P M$ modulation, a pulse position modulation $P P M$ symbol information of only one clock cycle is high, which contains a total of $2 \mathrm{M}$ clock cycles, only at the high level of the clock cycle to shine, therefore, a symbol of $P P M$ the average transmit optical power is:

$$
P_{P P M}=P_{1} / 2^{M}=P_{o o k} / 2^{M-1}
$$

Differential Pulse Position Modulation DPPM . In DPPM modulation, symbol contains a $D P P M\left(2^{M}+1\right) / 2$ clock cycles, only one clock cycle light, therefore, the average light power $D P P M$ is:

$$
P_{D P P M}=2 P_{1} /\left(2^{M}+1\right)=4 P_{\text {ook }} /\left(2^{M}+1\right)
$$

The Digital Pulse Interval Modulation DPIM . In DPIM modulation, a symbol containing DPIM $\left(2^{M}+3\right) / 2$ clock cycles, where only one clock cycle of light emission, and therefore, the average light power is DPIM:

$$
P_{\text {DPIM }}=2 P_{1} /\left(2^{M}+3\right)=4 P_{\text {ook }} /\left(2^{M}+3\right)
$$

\section{Conclusions}

All pulse time modulations are suitable for use in a wireless optical communication system. In this thesis, the current wireless optical communication field four common modulation technique: Off Keying OOK, pulse position modulation PPM, digital pulse interval modulation DPIM and differential pulse position modulation DPPM like. Through analysis, various modulation methods have their advantages and disadvantages, but from a practical point of view, mainly on account of slot bandwidth utilization and error rate. Therefore DPIM more advantages, more suitable for future atmospheric optical wireless communications.

\section{References}

[1] Zhanfang Zhou: Optical Communication Technology, Vol. 6 (2004) No 53, p.25-26

[2] Guoli Zhang: Optical Communication Technology, Vol. 12 (2005) No 27, p.74-76

[3] Hongtu Luo: Optical Communication Technology, Vol. 1 (2006) No 33, p.11-14

[4] Jieming Liu: Zhejiang University, Vol. 3 (2007) No33, p.121-124

[5] Qin Guo: Telecom Express, Vol. 1 (2008) No 23, p.56-63 